\title{
Deciphering the complex circulating immune cell microenvironment in chronic lymphocytic leukemia using patient similarity networks
}

\section{Zuzana Mikulkova}

Department of Immunology, Faculty of Medicine and Dentistry, Palacký University and University Hospital Olomouc, Czech Republic

\section{Gayane Manukyan}

Department of Immunology, Faculty of Medicine and Dentistry, Palacký University and University Hospital Olomouc, Czech Republic; Laboratory of Molecular and Cellular Immunology, Institute of Molecular Biology NAS RA, Yerevan, Armenia

\section{Peter Turcsanyi}

Department of Hematology-Oncology, Faculty of Medicine and Dentistry, Palacký University and University Hospital Olomouc, Czech Republic

\section{Renata Urbanova}

Department of Hematology-Oncology, Faculty of Medicine and Dentistry, Palacký University and University Hospital Olomouc, Czech Republic

\section{Jakub Savara}

Department of Computer Science, Faculty of Electrical Engineering and Computer Science, VSBTechnical University of Ostrava, Czech Republic

\section{Eliska Ochodkova}

Department of Computer Science, Faculty of Electrical Engineering and Computer Science, VSB-

Technical University of Ostrava, Czech Republic

\section{Milos Kudelka}

Department of Computer Science, Faculty of Electrical Engineering and Computer Science, VSBTechnical University of Ostrava, Czech Republic

\section{Yvona Brychtova}

Department of Internal Medicine, Hematology and Oncology, University Hospital Brno and Faculty of Medicine Masaryk University, Brno, Czech Republic

\section{Jan Molinsky}

1st Department of Medicine - Department of Hematology, First Faculty of Medicine, Charles University and General University Hospital in Prague, Praha, Czech Republic

\section{Martin Simkovic}

Department of Internal Medicine - Hematology, University Hospital and Medical School Hradec Kralove, Czech Republic 


\section{David Starostka}

Department of Clinical Hematology, Hospital in Havirov, Czech Republic

\section{Jan Novak}

Department of Internal Medicine - Hematology, Third Faculty of Medicine, Charles University and Faculty Hospital Kralovske Vinohrady, Praha, Czech Republic

\section{Ondrej Janca}

Department of Immunology, Faculty of Medicine and Dentistry, Palacký University and University Hospital Olomouc, Czech Republic

\section{Martin Dihel}

Department of Immunology, Faculty of Medicine and Dentistry, Palacký University and University Hospital Olomouc, Czech Republic

\section{Pavlina Ryznerova}

Department of Hematology-Oncology, Faculty of Medicine and Dentistry, Palacký University and University Hospital Olomouc, Czech Republic

\section{Lekaa Mohammad}

Department of Hematology and Oncology, Hospital in PIzen, Czech Republic

\section{Tomas Papajik}

Department of Hematology-Oncology, Faculty of Medicine and Dentistry, Palacký University and University Hospital Olomouc, Czech Republic

\section{Eva Kriegová ( $\sim$ eva.kriegova@email.cz)}

Lékařská Fakulta Univerzita Palackého v Olomouci https://orcid.org/0000-0002-8969-4197

\section{Research}

Keywords: Intermediate monocytes, Peripheral blood microenvironment, Patient similarity network, Immune cell activation, Novel agents

Posted Date: July 14th, 2020

DOI: https://doi.org/10.21203/rs.3.rs-41110/v1

License: (c) (1) This work is licensed under a Creative Commons Attribution 4.0 International License. Read Full License 


\section{Abstract}

Background: The tissue microenvironment in chronic lymphocytic leukemia (CLL) plays a key role in promoting neoplastic cell survival, proliferation, and drug resistance. There is a lack of complex characterization of CLL blood microenvironment and its clinical impact.

Methods: Immunophenotypic profiles of circulating immune cells in 244 CLL patients (untreated, $n=123$; novel agents, $n=67$; previous immunochemotherapy, $n=54)$ and age/sex-matched healthy controls $(n=52)$ were assessed using flow cytometry and analyzed by multivariate patient similarity networks (PSNs).

Results: Our study revealed high inter-individual heterogeneity in distribution and activation status of bystander immune cells in CLL, depending on the bulk of CLL cells. High CLL counts were associated with low activation status on circulating monocytes, $T$ and NK cells and vice versa low CLL counts with high activation of immune cells, reaching levels in controls. Regarding treatment, the highest activation of immune cells, particularly of intermediate and non-classical monocytes, was evident in patients treated with novel agents. Clustering and visualization using PSNs confirmed low activation of immune cells in progressive disease, irrespectively of IgHV status and Binet stage. Calculating time-to-event endpoint, patients with high intermediate monocytes $(>5.4 \%)$, predominantly low activated, were associated with 2.5 -fold higher likelihood $(95 \% \mathrm{Cl} 1.421-4.403, \mathrm{P}=0.002)$ of event than those with low percentage of intermediate monocytes.

Conclusions: Activation of circulating immune cells are dependent on the CLL cell counts and used therapy, with the lowest activation in patients with progressive disease. Percentage and activation of intermediate monocytes could be of prognostic value in CLL.

\section{Background}

Chronic lymphocytic leukemia (CLL) is a lymphoproliferative disorder characterized by the progressive accumulation of mature CD19 + CD $5+$ B-cells in the peripheral blood, bone marrow and secondary lymphoid organs [1]. CLL is characterized by extremely variable clinical course, ranging from an indolent and stable disease without a requirement for therapy to a highly aggressive disease requiring treatment.

While certain "intrinsic factors", mainly genomic alterations of CLL cells, have been shown crucially involved in CLL pathogenesis, the effect of "extrinsic factors" such as direct interactions of CLL cells within the microenvironment are not fully estimated. In fact, CLL is highly dependent on bidirectional crosstalk between CLL cells and a supportive microenvironment allowing CLL cells to escape from immunosurveillance [2,3]. Many cellular and molecular players in the tissue microenvironment promote survival, proliferation and resistance of CLL cells, including monocyte-derived nurse-like cells, monocytederived macrophages, and mesenchymal stromal cells [2,4]. In addition, the critical importance of the tissue microenvironment is supported by the clinical effects of novel agents that disrupt the interaction between neoplastic cells and the tissue microenvironment $[5,6,7]$. Compared to the tissue microenvironment, the complex architecture of the circulating blood microenvironment in the CLL has not 
yet been described. Studies focused on individual cell populations in blood reported disturbances in the activation and exhaustion of T cells [8,9], neutrophils [10], decreased degranulation, and increased sensitivity to activation-induced NK cell death [11], as well as altered composition and phagocytosis of monocytes in patients with CLL $[12,13]$. Comprehensive studies on circulating immune cells and their cross-talk with neoplastic CLL cells may contribute to new insights into the complexity of tumor immunology in CLL.

We, therefore, aimed to comprehensively characterize circulating immune cells that shape CLL blood microenvironment together with CLL cells in a large real-world cohort of CLL patients from seven Czech centers. Moreover, we addressed a question, whether blood immune cells and their activation differ between patients with various tumor burden, clinical course, and used treatment, with special emphasis to novel drugs.

\section{Materials And Methods}

\section{Patients}

Peripheral blood samples were collected from 244 patients with CLL (F/M: 105/139, median age 67 years) from seven hematology-oncology centers in the Czech Republic; the diagnosis of CLL was established according to the IWCLL guidelines [14]. Of enrolled patients, 123 patients were treatmentnaïve (TN), 67 patients were treated with novel agents (ibrutinib, idelalisib, venetoclax) and 54 patients were previously receiving immunochemotherapy. Detailed clinical characteristic of enrolled patients is shown in Table 1. Time-to-event endpoint (TTE) was available for all patients during one-year of postsampling follow-up. The study included 52 age-matched healthy control subjects (F/M: 28/24, median age 68 years) selected from members of medical staff or their relatives, where cancer, autoimmunity and chronic diseases were excluded by the questionnaires.

All CLL patients and healthy controls provided written informed consent for the use of peripheral blood for research purposes in accordance with the Declaration of Helsinki. The local ethical committee of the University Hospital and Palacký University Olomouc approved the study. 
Table 1

Characteristics of CLL patients

\begin{tabular}{|ll|}
\hline Parameters & $\begin{array}{l}\text { CLL patients } \\
(\mathbf{n}=\mathbf{2 4 4})\end{array}$ \\
\hline Age in years, median (range) & $67(38-89)$ \\
\hline Gender, Female/Male & $105 / 139$ \\
\hline Binet stage, A/B/C/n.a. & $143 / 54 / 44 / 3$ \\
\hline Bulky lymphadenopathy $\geq 5$ cm, Yes/No/n.a. & $75 / 158 / 11$ \\
\hline Splenomegaly, Yes/No/n.a. & $88 / 148 / 8$ \\
\hline IgHV mutational status, Unmutated/Mutated/n.a. & $150 / 83 / 11$ \\
\hline B2-microglobulin ( $\geq 3.5$ mg/dL), Yes/No/n.a. & $116 / 109 / 19$ \\
\hline Genetic characteristics at sampling & \\
\hline TP53 disruption (deletion 17p and/or TP53 mutations), Yes/No/n.a. & $59 / 183 / 2$ \\
\hline del(11q22), Yes/No/n.a. & $55 / 189 / 0$ \\
\hline del(13q14), Yes/No/n.a. & $112 / 81 / 51$ \\
\hline Treatment history, Yes/No & $121 / 123$ \\
\hline Patients previously treated with immunochemotherapy & 54 \\
\hline Number of previous treatment regimens, median (range) & $1(1-4)$ \\
\hline Time from the last treatment to sampling, median (range) in months & $24(1-173)$ \\
\hline Patients treated with novel drugs & 67 \\
\hline Ibrutinib/idelalisib/venetoclax & $42 / 19 / 6$ \\
\hline Number of previous treatment regimens, median (range) & $2(0-10)$ \\
\hline Treatment duration on novel drugs, median (range) in months & $9(1-42)$ \\
\hline CLL cells in peripheral blood & $68.6(0.01-98.9)$ \\
\hline Percentage, mean (min-max) & $59.7(0.01-592)$ \\
\hline CLL cell counts (x10 $/$ L), mean (min-max) & \\
\hline n.a. - not available & \\
\hline
\end{tabular}

\section{Flow cytometry}

Six-color flow cytometry was used to analyze the circulating immune cells and their main activation markers (on-line supplement Table S1) in the whole blood as reported previously [10, 13]. Briefly, whole 
blood was incubated with the mix of conjugated antibodies for 20 min in the dark at room temperature. Then, the red blood cells were lysed with $2 \mathrm{~mL}$ of FACS lysing solution (diluted 1:10 with distilled water; Becton-Dickinson, San Jose, CA, USA) and washed with phosphate-buffered saline. The gating strategy for investigated blood populations is described elsewhere [15]. Briefly, the main cell populations were identified using a sequential gating strategy after the exclusion of doublets (FSC-A versus FSC-H) as follows: CLL cells (CD5+/CD19+), CD4 + T cells (CD3+/CD4+), CD8 + T cells (CD3+/CD8+), Treg cells (CD4+/CD25+/CD127-), NK cells (CD3-/CD16+/CD56+), neutrophils (CD15+/CD16+), and monocytes (CD14+). Monocyte subsets were gated as follows: classical (CD14+/CD16-), intermediate (CD14+/CD16+), and non-classical (CD14dim/CD16+). The complete list of used monoclonal antibodies (all BioLegend, San Diego, CA, USA) is displayed in on-line supplement Table S2. Isotype matched FITC, PE, PerCP-Cy5.5, PE-Cy7, APC, APC-Cy-7-conjugated irrelevant antibodies (all MOPC-21, BioLegend) were used as negative controls. The analysis was performed on a flow cytometer BD FACSCanto II (Becton Dickinson). Flow cytometry data were analyzed using the FlowJo vX0.7 software (Tree Star, Inc, San Carlos, CA, USA).

The main populations (lymphocytes, neutrophils, monocytes) were calculated as a percentage of immune cell singlets. Percentage of subpopulations was calculated as a part of parental population: CLL from lymphocytes, CD4 + and CD8 + T cells from CD3 + T cells, Treg cells from CD $4+T$ cells, classical/intermediate/non-classical subsets from monocytes, respectively.

CLL and immune cell counts were expressed as the number of cells per unit of volume (cells/L) and were calculated by multiplying a percentage of cells derived from flow cytometry by the number of white blood cells from a complete blood count. Studied subpopulations and markers were expressed as a percentage of positive cells and median fluorescence intensity (MFI).

\section{Statistics and Patient similarity network analysis (PSN)}

Statistical tests (non-parametric Mann-Whitney-Wilcoxon test, Spearman's Rank-Order Correlations, generation of Receiver Operating Curves (ROC), Kaplan-Meier curves, and odds ratio (OR) were performed using R statistical software (http://www.r-project.org/). P-values of $<0.05$ were considered significant.

For the clustering of patients and visualization of patient similarities on the basis of circulating cell populations, a method of network construction based on the nearest neighbor analysis was applied [16, $17,18]$. Briefly explained, the data on activation of individual cell populations and/or their counts were transferred to the network, followed by automatic detection of clusters in the network [19]. To convert the dataset to a weighted undirected network, the LRNet method [16] based on nearest-neighbors analysis was used (https://homel.vsb.cz/ kud007//rnet_files/). Nodularity and silhouettes were used to assess the quality of obtained clusters. For calculation of trend of changes across particular clusters see On-line supplement.

\section{Results}




\section{Patient similarity networks (PSNs) analysis visualized the relationship between activation status of immune cells and CLL cell counts}

Multivariate PSNs were used to cluster patients with similar profile regarding the CLL counts and activation status of immune cells. Of investigated cells (CD4 + and CD8 + T lymphocytes, Tregs, NK cells, neutrophils, classical/intermediate/non-classical monocytes, and CLL cells) and their activation markers, the best clustering of patients was observed using CLL counts and HLA-DR expression on CLL cells, classical, intermediate and non-classical monocyte subsets. As shown in Fig. 1, CLL patients were clustered into four clusters showing the inverse relationship between the CLL cell counts and activation of CLL and monocyte subsets. Generally, high percentage and CLL cell counts were associated with a low activation status on CLL cells and all monocyte subsets and vice versa low CLL cell counts with high activation of CLL cells and classical, intermediate and non-classical monocyte subsets (Fig. 1B).

This observation was confirmed by correlation analysis, which revealed strong negative correlation of the CLL cell counts with HLA-DR and CD64 expression on classical and intermediated monocytes, and HLADR on non-classical monocytes, CD4 + and CD8 + T cells in our CLL patients $(P<0.001)$ (Fig. 2, on-line supplement Table S3). Additionally, CLL counts correlated positively with Tregs and CD4+/CD8 + ratio (both $P<0.001)$. The number of CLL cells did not correlate with the number of classical monocytes $(P=$ $0.480)$, intermediate monocytes $(P=0.880)$ as well as non-classical monocytes $(P=0.120)$, respectively (on-line supplement Table S3).

\section{Patient similarity networks (PSNs) analysis based on activation markers of immune cells in CLL patients}

In order to investigate the activation of immune cells and its association with clinical parameters and treatment regimes, we created PSNs by combining all activation markers of all immune subpopulations, not taking into account the CLL cell counts. Patients on novel drugs such as ibrutinib (an irreversible inhibitor of Bruton's tyrosine kinase, BCR inhibitor), idelalisib (an inhibitor of the $\delta$ isoform of phosphatidylinositol 3-kinase, BCR inhibitor) and venetoclax (BCL2 inhibitor) were compared with TN patients as well as patients pretreated with immunochemotherapy. PSNs revealed four patient clusters based on similarities on HLA-DR expression on subsets of monocytes (classical, intermediate, nonclassical), CD4 + and CD8 + lymphocytes, and NK cells (on-line supplement Figure S1). The trend of changes in the activation of a particular cell population(s) across the clusters is shown in Fig. 3A.

For better visualization of the relationship between immune cell activation and the number of CLL cell, IgHV mutational status, and treatment regime, respectively, the dots corresponding to individual patients were colored based on the data of each particular patient. As visible in Fig. 3, CLL patients with highly activated immune cells had predominantly low counts of CLL cells and were enriched within treatmentnaïve (TN) patients with indolent disease course. TN patients who required treatment within follow-up 
time were associated with the clusters of less activated immune cells (Fig. 3E, on-line supplement text and Tables S4, S5).

CLL patients treated with novel agents showed high activation of all immune cells, irrespective of the type of drug (Fig. 3C, 3D). Higher activation of immune cells on novel drugs was also confirmed on a subset of patients with an equal number of CLL cells treated with novel drugs and previous immunochemotherapy. Increased HLA-DR expression on intermediate $(P=0.012)$ and non-classical $(P=0.028)$ monocytes, decreased percentage of non-classical monocytes $(P=0.046)$, classical monocytes $(P=0.038)$, and Treg cells $(P<0.001)$ were detected in patients treated with novel agents (on-line supplement Table S5).

The patients with mutated and unmutated $I g H V$ status were distributed across all clusters, irrespective of the activated status of immune cells (Fig. 3F).

\section{Time-dependent changes of HLA-DR on classical monocytes during long-term treatment with ibrutinib and idelalisib}

Next, we were interested whether activation of immune cells differs between CLL patients treated with two BCR inhibitors: ibrutinib and idelalisib. Moreover, we investigated the time-dependent dynamics of changes in the activation of immune cells of CLL patients on both drugs.

Firstly, we analyzed the expression of surface markers (CD27, CD38, CD49d and HLA-DR) on CLL cells in patients treated with ibrutinib and idelalisib and revealed no difference in analyzed markers between these patient groups. Regarding immune cells, increased percentage of intermediate monocytes $(P=$ 0.002), as well as their expression of HLA-DR $(P=0.007)$ and $\operatorname{CD} 64(P=0.018)$, but not their cell counts, were detected in patients treated with idelalisib comparing to ibrutinib, irrespectively of treatment duration (Fig. 4, on-line supplement Table S6).

Analysis of longitudinal samples from patients treated with ibrutinib and idelalisib $(n=11)$ showed changes in activation markers on immune cells, as well as the number of CLL cells during the treatment course, with marked differences between ibrutinib and idelalisib. In line with previous studies [20, 21], the marked increase in the CLL cell counts within two months after ibrutinib treatment initiation was observed in majority of patients followed by a decline to almost zero values during the first year of treatment (Fig. 4A). Simultaneously with the decrease in the CLL count, the trend of increasing HLA-DR expression on classical and intermediate monocytes (Fig. 4A) as well as non-classical monocytes, CD4 + and CD $8+T$ cells (data not shown) was detected during the first year of ibrutinib treatment, but not on idelalisib. Expression of CD64 on intermediated monocytes dropped in the first few months, followed by stable levels (Fig. 4A). In idelalisib treated patients, a decrease in the number of CLL cells was evident during the first months after the treatment initiation, whereas the expression of HLA-DR and CD64 on monocytes fluctuated during the follow-up. Compared to ibrutinib treatment, there was a trend to increased CD64 expression in idelalisib-treated patients in the first months after starting treatment (Fig. 4B). 


\section{HLA-DR expression on monocyte subpopulations does not differ between treatment-naïve (TN) CLL patients and healthy controls}

Next, we analyzed the differences in activation of CLL and immune cells in TN patients who required treatment $(n=50)$ within 1 year after sampling and TN patients with the indolent course $(n=73)$.

Moreover, all studied parameters were compared with those on CD19 + B cells from age- and sex-matched healthy subjects.

TN patients who required treatment during follow-up exhibited higher percentage as well as number of CLL cells (both $P<0.001)$ and elevated percentage of intermediate monocytes $(P<0.001)$ comparing to TN patients with the indolent course (median follow-up 26 months). When comparing TN CLL patients with healthy controls, the TN patients with indolent course did not differ from healthy controls in terms of activation of monocytes subsets (Fig. 5). The TN patients requiring treatment within 1-year follow-up time had lower activation on all monocyte subsets comparing to healthy controls. In all TN patients, a higher proportion of Treg cells $(P<0.001)$ was detected in comparison to healthy controls.

\section{Percentage of intermediate monocytes is associated with time-to-event endpoint}

To estimate the association of circulating immune cells and/or their activation with time-to-event endpoint (TTE), we performed ROC analysis in newly diagnosed patients ( $n=34$, derivation cohort) subdivided according to the treatment requirement/death during one-year post-sampling follow-up. Of the studied markers and cell counts, percentage of intermediate monocytes (cut-off 5.4\%) achieved the most discriminatory power reaching area under the ROC curve (AUC) of 0.836 (Fig. 6A). Similar results were obtained when analyzing newly diagnosed TN patients subdivided according to the low ( $<4$ points) vs high ( $\geq 4$ points) CLL-IPI prognostic score (AUC $=0.805$, data not shown). Moreover, patients with a low percentage $(<5.4 \%)$ of intermediate monocytes had higher expression of HLA-DR than those with a high percentage of intermediate monocytes $(P=0.020$, on-line supplement Figure S2). Regarding the activation markers, HLA-DR expression on intermediate monocytes $(A U C=0.814)$ and classical monocytes $(A U C=0.764)$ also showed high discriminatory power for TTE.

Furthermore, Kaplan-Meier (K-M) curves were generated in CLL patient subgroups subdivided according to the percentage of intermediate monocytes (cut-off $5.4 \%)$ in the derivation $(n=34)$ and validation cohorts $(n=210)$, respectively. The K-M curves showed that CLL patients with more than $5.4 \%$ of intermediate monocytes have a shorter TTE than those with a lower percentage of intermediate monocytes in both derivation ( $P=0.020$, Fig. 6B) and validation cohorts $(P=0.002$, Fig. $6 \mathrm{C})$, respectively. Odds ratio (OR) analysis showed that CLL patients with higher intermediate monocytes $(>5.4 \%)$, which were predominantly low activated, have 2.5 -fold higher likelihood $(95 \% \mathrm{Cl} 1.421-4.403, P=0.002)$ of event within one-year follow-up than those with low percentage of intermediate monocytes. 


\section{Discussion}

This first study on the complex characterization of the blood microenvironment in CLL showed that activation of circulating monocytes, T and NK cells is associated with CLL cell counts and therapy used, with the lowest activation seen in patients with progressive disease and the highest activation in patients with indolent untreated disease and those treated with novel agents.

The dependency of CLL cells on signals from the microenvironment in the bone marrow and lymph nodes is widely accepted [2,3], but the CLL cell cross-talk within the blood immune microenvironment is not yet fully characterized. We, therefore, investigated the blood immune cell microenvironment in terms of heterogeneity and activation of the immune cells and its relationship to the number of CLL cells. Our data revealed a dominant association for the bulk of CLL cells with the activation status of blood microenvironment. Increased number of CLL cells was associated with the decreased HLA-DR expression by monocyte subsets, T cells, and CLL cells themselves. And opposite, lower number of CLL cells was associated with the elevation of HLA-DR on CLL cells as well as almost all immune cell subsets in circulation, reaching levels in healthy controls. HLA-DR is considered to be a very late activation marker on the surface of monocytes, macrophages, NK cells, B and T lymphocytes [22, 23]. In the context of cancer biology, HLA-DR has been reported to serve as a favorable prognostic marker for several cancers such as colorectal carcinoma [24], glioma [25], and melanoma [26]. Moreover, HLA-DR expression on leukemic cells was suggested to be essential for establishing effective antitumor immunity via CD4 + cell activation and the recruitment of effector cell populations, such as macrophages [27]. Besides being an important prognostic marker on tumor cells, HLA-DR expression on immune cells has been shown to reflect the tumor immune status and the response to the therapy [28]. In line with the above evidence, we suggested that HLA-DR expression may reflect disease progression and the treatment response in CLL as well. To support this hypothesis, low activation of monocyte subpopulations was observed in a group of untreated patients requiring treatment during a one-year follow-up. Conversely, patients with higher activation of monocyte subpopulations, particularly intermediate monocytes, were TN patients with an indolent disease course. The crucial impact of CLL bulk on blood microenvironment observed in our study is also supported by a recent study showing that CLL cells impair mitochondrial fitness in CD $8+T$ cells and impede CAR T-cell efficacy [29]. Also other studies showed that CLL cells are active participants in microenvironmental cross-talk [30] contributing to the inhibition of effective immune cell activation by production of spectrum of immunosuppressive mediators [12,31, 32], most prominently in the lymph nodes [31]. Here we provide evidence of the immunosuppressive condition also in circulation in CLL. The exact mechanism by which the CLL suppress the activation of bystander immune cells needs to be investigated in future studies.

Because our multivariate analysis revealed a significant relationship between the activation of immune cells and disease course, we explored their prognostic value. We showed that the percentage of intermediate and classical monocytes and their activation have a prognostic potential for the time-toevent endpoint. In particular, a high percentage of intermediate monocytes (> 5.4\%) was prognostic for progressive disease and these patients have 2.5 -fold higher likelihood of event than those with low 
percentage of intermediate monocytes, irrespective of the treatment. The importance of monocytes for the prognosis of CLL patients has been demonstrated in recent studies showing association of monocyte counts, particularly of circulating classical monocytes, with time to the first therapy, aggressiveness and disease outcome [33,34]. Regarding monocytes and monocyte-derived cells in CLL, there is already evidence that they promote CLL cell survival and proliferation [34], and have deregulated genes involved in phagocytosis and inflammation [12]. Key role of monocytes in CLL is also supported by observations in animal model of CLL, where depletion of monocytes and macrophages resulted in a control of CLL development and reparation of immune dysfunction in vivo [35]. The exact mechanisms by which monocytes and particularly intermediate monocytes contribute to the pathogenesis of CLL need to be clarified in future studies.

Despite our study showed the dependency of the activation of blood bystander cells on the bulk of CLL cells, no correlation was detected with other prognostic markers for CLL such as IgHV status, Binet stage, $\beta$-2-microglobulin, TP53 disruption, CD49d, and CD38. It seems that these prognostic markers do not have direct impact in the establishment of immune microenvironment, but have an influence on CLL cell counts and correspondingly on the disease course, the survival time, and risk-adapted treatment options, as it is shown by others $[7,36,37]$. Besides genetic makeup emerging concept, our data highlights the CLL cells as an additional determinant of the immune contexture in CLL. It remains to clarify the causal link between genetic landscape, immunophenotype and cytokines/chemokines produced by CLL cells and bystander immune cells and their function and behavior, factors influencing the disease course as well as results of the intervention strategies.

Next, we were interested in the changes of blood microenvironment in patients treated with ibrutinib, idelalisib and venetoclax that have recently emerged as highly promising novel therapeutic strategies in $\operatorname{CLL}[5,6,7]$. Although these drugs target predominantly $\operatorname{CLL}$ cells $[5,6,7,38]$, there is growing evidence of their effects on the microenvironment. Ibrutinib and idelalisib have been shown to have an immunomodulatory effect on T cells [5, 13, 39, 40,41,42], NK cells [43], monocytes [13], and production of Th2 cytokines [44], depending on the time of treatment [13]. Also venetoclax has been shown to modulate number and activation of $\mathrm{T}$ and $\mathrm{B}$ cells, restore NK cell function and reduce overproduction of inflammatory cytokines [45]. In our study, multivariate analyses revealed activation of all circulating immune cells in the majority of patients treated with novel drugs. Importantly, the activation of all immune cells in the majority of our patients receiving novel drugs was more pronounced compared with those previously treated with immunochemotherapy or untreated patients, even after correction for CLL cell number. In line with our data, a recent study using venetoclax-based regimens revealed a reduction in the immunosuppressive footprint of CLL, suggesting that immune system regeneration occurs after removal of leukemic cells [45]. Similarly, ibrutinib has been shown to modulate the immunosuppressive CLL microenvironment, through STAT3-mediated suppression of regulatory B-cell function and inhibition of the PD-1/PD-L1 pathway [46]. Our data further supports the key contribution of novel agents to the immunomodulation of circulating microenvironment in CLL. The exact mechanism of action of the novel agents on the microenvironment needs to be elucidated, while both the indirect effect due to the elimination of neoplastic cells and the direct activation of immune cells can be expected. Interestingly, 
some our patients on novel agents had a very low HLA-DR expression on immune cells. Since HLA-DR expression on immune cells may reflect the response to the therapy as shown in breast cancer patients [28], our patients on novel drugs with low immune cell activation may have a high risk of disease progression, as shown in some cases. Interestingly, our data showed different time-dependent dynamics of immune cell activation and redistribution of CLL cells from tissues to peripheral blood when treated with ibrutinib and idelalisib, respectively. Our data points to the critical impact of the treatment regimens on both drugs not only on abrogation of BCR-dependent survival and proliferation signals but also on activation of immune cells and establishing of non-tumor cells functionality.

Our study has some limitations. Firstly, we analyzed real-world CLL cohort of patients sampled at different time points and treatment regimens and only several patients had longitudinal samples. Secondly, we did not have paired lymph node or bone marrow samples to examine these microenvironments simultaneously. Third, we did not perform a detailed characterization of the underlying immune response and functional investigations that should be performed in future studies. Despite these limitations, we believe that this first study on deciphering the complex circulating microenvironment by advanced multivariate analysis helped to highlight the critical contribution of immune cells to the disease outcome and treatment response in CLL.

\section{Conclusions}

In summary, we provide evidence that the shaping of peripheral blood immune cells is strongly dependent on the bulk of CLL cells. Our findings highlight the role of monocytes, particularly of intermediate monocytes, in the pathogenesis of CLL and nominate them as an independent prognostic marker and potentially as a therapeutic target in CLL. Assessment of responsiveness to the therapy based on the profile of immune cells may represent a next step in improving the success of current immunotherapies and the development of next-generation novel drugs.

\section{Abbreviations}

AUC

Area under the ROC Curve

Bcl-2

B-cell lymphoma 2

BCR

B-cell receptor

CLL

Chronic lymphocytic leukemia

\section{CLL-IPI}

CLL-International prognostic index

FSC-A/-H

Forward scatter-Area/-Height 
IgHV

Immunoglobulin heavy chain variable region

$\mathrm{K}-\mathrm{M}$ curve

Kaplan-Meier curve

MFI

Median fluorescence intensity

MON

Monocytes

n.a.

Not available

OR

Odds ratio

PSN

Patient similarity network

ROC

Receiver operating curves

TN

Treatment-naïve

TTE

Time-to-event endpoint

\section{Declarations}

\section{Ethics approval and consent to participate}

This study was approved by ethical committee of the University Hospital and Palacký University Olomouc. All CLL patients and healthy controls provided written informed consent for the use of peripheral blood for research purposes in accordance with the Declaration of Helsinki.

\section{Consent for publication}

Not applicable.

\section{Availability of data and materials}

The data and materials of this study are available from the corresponding author on reasonable request.

\section{Competing interests}


Authors declare no competing financial interests in relation to the work described.

\section{Funding}

This work was supported by a grant of Ministry of Health of the Czech Republic (FNOL, 00098892) and IGA_LF UP_2020_16.

\section{Authors' contributions}

$\mathrm{ZM}, \mathrm{GM}$, and EK conceived the study, interpreted the results and wrote the manuscript. ZM, GM, OJ, and MD performed the experiments. ZM, JS, EO, and MK analyzed the data. PT, RU, YB, JM, MS, DS, JN, PR, $L M$, and TP collected the patient's samples and clinical data. TP critically revised the manuscript. All authors edited and approved the final version of the manuscript.

\section{Acknowledgements}

Not applicable.

\section{References}

1. Zhang S, Kipps TJ. The pathogenesis of chronic lymphocytic leukemia. Annu Rev Pathol. 2014;9:103-18.

2. Ten Hacken E, Burger JA. Microenvironment interactions and B-cell receptor signaling in Chronic Lymphocytic Leukemia: Implications for disease pathogenesis and treatment. Biochim Biophys Acta. 2016;1863(3):401-13.

3. van Attekum $\mathrm{MH}$, Eldering E, Kater AP. Chronic lymphocytic leukemia cells are active participants in microenvironmental cross-talk. Haematologica. 2017;102(9):1469-76.

4. Herishanu Y, Pérez-Galán P, Liu D, Biancotto A, Pittaluga S, Vire B, et al. The lymph node microenvironment promotes B-cell receptor signaling, NF-kappaB activation, and tumor proliferation in chronic lymphocytic leukemia. Blood. 2011;117(2):563-74.

5. Niemann CU, Herman SE, Maric I, Gomez-Rodriguez J, Biancotto A, Chang BY, et al. Disruption of in vivo Chronic Lymphocytic Leukemia Tumor-Microenvironment Interactions by Ibrutinib - Findings from an Investigator-Initiated Phase II Study. Clin Cancer Res. 2016;22(7):1572-82.

6. Schiattone L, Ghia P, Scarfò L. The evolving treatment landscape of chronic lymphocytic leukemia. Curr Opin Oncol. 2019;31(6):568-73.

7. Hallek M. Chronic lymphocytic leukemia: 2020 update on diagnosis, risk stratification and treatment. Am J Hematol. 2019;94(11):1266-87. 
8. Riches JC, Davies JK, McClanahan F, Fatah R, Iqbal S, Agrawal S, et al. T cells from CLL patients exhibit features of T-cell exhaustion but retain capacity for cytokine production. Blood. 2013;121:1612-21.

9. Palma M, Gentilcore G, Heimersson K, Mozaffari F, Näsman-Glaser B, Young E, et al. T cells in chronic lymphocytic leukemia display dysregulated expression of immune checkpoints and activation markers. Haematologica. 2017;102(3):562-72.

10. Manukyan G, Papajik T, Gajdos P, Mikulkova Z, Urbanova R, Gabcova G, et al. Neutrophils in chronic lymphocytic leukemia are permanently activated and have functional defects. Oncotarget. 2017;8:84889-901.

11. MacFarlane AW 4th, Jillab M, Smith MR, Alpaugh RK, Cole ME, Litwin S, et al. NK cell dysfunction in chronic lymphocytic leukemia is associated with loss of the mature cells expressing inhibitory killer cell Ig-like receptors. Oncoimmunology. 2017;6(7):e1330235.

12. Maffei R, Bulgarelli J, Fiorcari S, Bertoncelli L, Martinelli S, Guarnotta C, et al. The monocytic population in chronic lymphocytic leukemia shows altered composition and deregulation of genes involved in phagocytosis and inflammation. Haematologica. 2013;98(7):1115-23.

13. Manukyan G, Turcsanyi P, Mikulkova Z, Gabcova G, Urbanova R, Gajdos P, et al. Dynamic changes in HLA-DR expression during short-term and long-term ibrutinib treatment in patients with chronic lymphocytic leukemia. Leuk Res. 2018;72:113-9.

14. Hallek M, Cheson BD, Catovsky D, Caligaris-Cappio F, Dighiero G, Döhner H, et al. Guidelines for the diagnosis and treatment of chronic lymphocytic leukemia: a report from the International Workshop on Chronic Lymphocytic Leukemia updating the National Cancer Institute-Working Group 1996 guidelines. Blood. 2008;111:5446-56.

15. Rühle PF, Fietkau R, Gaipl US, Frey B. Development of a Modular Assay for Detailed Immunophenotyping of Peripheral Human Whole Blood Samples by Multicolor Flow Cytometry. Int J Mol Sci. 2016;17(8):pii: E1316.

16. Ochodkova E, Zehnalova S, Kudelka M. Graph construction based on local representativeness. In: Cao Y, Chen J, editors. Computing and Combinatorics. COCOON 2017. Lecture Notes in Computer Science. Cham: Springer; 2017. pp. 654-65.

17. Kriegova E, Manukyan G, Mikulkova Z, Gabcova G, Kudelka M, Gajdos P, et al. Gender-related differences observed among immune cells in synovial fluid in knee osteoarthritis. Osteoarthritis Cartilage. 2018;26(9):1247-56.

18. Gallo J, Kriegova E, Kudelka M, Lostak J, Radvansky M. Gender Differences in Contribution of Smoking, Low Physical Activity, and High BMI to Increased Risk of Early Reoperation After TKA. J Arthroplasty. 2020;35:1545-57.

19. Blondel VD, Guillaume JL, Lambiotte R, Lefebvre E. Fast unfolding of communities in large networks. J Stat Mech Theory Exp. 2008;2008:P10008.

20. Herman SE, Niemann CU, Farooqui M, Jones J, Mustafa RZ, Lipsky A, et al. Ibrutinib-induced lymphocytosis in patients with chronic lymphocytic leukemia: correlative analyses from a phase II 
study. Leukemia. 2014;28(11):2188-96.

21. Woyach JA, Smucker K, Smith LL, Lozanski A, Zhong Y, Ruppert AS, et al. Prolonged lymphocytosis during ibrutinib therapy is associated with distinct molecular characteristics and does not indicate a suboptimal response to therapy. Blood. 2014;123(12):1810-17.

22. Reddy M, Eirikis E, Davis C, Davis HM, Prabhakar U. Comparative analysis of lymphocyte activation marker expression and cytokine secretion profile in stimulated human peripheral blood mononuclear cell cultures: an in vitro model to monitor cellular immune function. J Immunol Methods. 2004;293(1-2):127-42.

23. Saez-Cirion A, Lacabaratz C, Lambotte O, Versmisse P, Urrutia A, Boufassa F, et al. HIV controllers exhibit potent CD8 T cell capacity to suppress HIV infection ex vivo and peculiar cytotoxic T lymphocyte activation phenotype. Proc Natl Acad Sci USA. 2007;104(16):6776-81.

24. Sconocchia G, Eppenberger-Castori S, Zlobec I, Karamitopoulou E, Arriga R, Coppola A, et al. HLA class II antigen expression in colorectal carcinoma tumors as a favorable prognostic marker. Neoplasia. 2014;16(1):31-42.

25. Diao J, Xia T, Zhao H, Liu J, Li B, Zhang Z. Overexpression of HLA-DR is associated with prognosis of glioma patients. Int J Clin Exp Pathol. 2015;8(5):5485-90.

26. Johnson DB, Estrada MV, Salgado R, Sanchez V, Doxie DB, Opalenik SR, et al. Melanoma-specific $\mathrm{MHC}$-II expression represents a tumour-autonomous phenotype and predicts response to anti-PD1/PD-L1 therapy. Nat Commun. 2016;7:10582.

27. Corthay A, Skovseth DK, Lundin KU, Røsjø E, Omholt H, Hofgaard PO, et al. Primary antitumor immune response mediated by CD4 + T cells. Immunity. 2005;22(3):371-83.

28. Saraiva DP, Jacinto A, Borralho P, Braga S, Cabral MG. HLA-DR in Cytotoxic T Lymphocytes Predicts Breast Cancer Patients' Response to Neoadjuvant Chemotherapy. Front Immunol. 2018;9:2605.

29. van Bruggen JAC, Martens AWJ, Fraietta JA, Hofland T, Tonino SH, Eldering E, et al. Chronic lymphocytic leukemia cells impair mitochondrial fitness in CD8 + T cells and impede CAR T-cell efficacy. Blood. 2019;134(1):44-58.

30. van Attekum MH, Eldering E, Kater AP. Chronic lymphocytic leukemia cells are active participants in microenvironmental cross-talk. Haematologica. 2017;102(9):1469-76.

31. de Weerdt I, Hofland T, de Boer R, Dobber JA, Dubois J, van Nieuwenhuize D, et al. Distinct immune composition in lymph node and peripheral blood of CLL patients is reshaped during venetoclax treatment. Blood Adv. 2019;3(17):2642-52.

32. Arruga F, Gyau BB, lannello A, Vitale N, Vaisitti T, Deaglio S. Immune Response Dysfunction in Chronic Lymphocytic Leukemia: Dissecting Molecular Mechanisms and Microenvironmental Conditions. Int J Mol Sci. 2020;21(5):pii: E1825.

33. Lapuc I, Bolkun L, Eljaszewicz A, Rusak M, Luksza E, Singh P, et al. Circulating classical CD14 + + CD16- monocytes predict shorter time to initial treatment in chronic lymphocytic leukemia patients: Differential effects of immune chemotherapy on monocyte-related membrane and soluble forms of CD163. Oncol Rep. 2015;34(3):1269-78. 
34. Friedman DR, Sibley AB, Owzar K, Chaffee KG, Slager S, Kay NE, et al. Relationship of blood monocytes with chronic lymphocytic leukemia aggressiveness and outcomes: a multi-institutional study. Am J Hematol. 2016;91(7):687-91.

35. Hanna BS, McClanahan F, Yazdanparast H, Zaborsky N, Kalter V, Rößner PM, et al. Depletion of CLLassociated patrolling monocytes and macrophages controls disease development and repairs immune dysfunction in vivo. Leukemia. 2016;30(3):570-9.

36. Eichhorst B, Hallek M. Prognostication of chronic lymphocytic leukemia in the era of new agents. Hematology Am Soc Hematol Educ Program. 2016;2016(1):149-55.

37. Boddu P, Ferrajoli A. Prognostic Factors in the Era of Targeted Therapies in CLL. Curr Hematol Malig Rep. 2018;13(2):78-90.

38. Sedlarikova L, Petrackova A, Papajik T, Turcsanyi P, Kriegova E. Resistance-associated mutations in chronic lymphocytic leukaemia patients treated with novel agents. Front Oncol. 2020;10:894.

39. Dubovsky JA, Beckwith KA, Natarajan G, Woyach JA, Jaglowski S, Zhong Y, et al. Ibrutinib is an irreversible molecular inhibitor of ITK driving a Th1-selective pressure in T lymphocytes. Blood. 2013;122(15):2539-49.

40. Martinelli S, Maffei R, Fiorcari S, Quadrelli C, Zucchini P, Benatti S, et al. Idelalisib impairs T-cellmediated immunity in chronic lymphocytic leukemia. Haematologica. 2018;103(12):e598-601.

41. Parry HM, Mirajkar N, Cutmore N, Zuo J, Long H, Kwok M, et al. Long-Term Ibrutinib Therapy Reverses CD8 + T Cell Exhaustion in B Cell Chronic Lymphocytic Leukaemia. Front Immunol. 2019;10:2832.

42. Long M, Beckwith $K$, Do P, Mundy BL, Gordon A, Lehman AM, et al. Ibrutinib treatment improves $T$ cell number and function in CLL patients. J Clin Invest. 2017;127(8):3052-64.

43. Kohrt HE, Sagiv-Barfi I, Rafiq S, Herman SE, Butchar JP, Cheney C, et al. Ibrutinib antagonizes rituximab-dependent NK cell-mediated cytotoxicity. Blood. 2014;123:1957-60.

44. Pleyer $C$, Wiestner A, Sun C. Immunological changes with kinase inhibitor therapy for chronic lymphocytic leukemia. Leuk Lymphoma. 2018;59(12):2792-800.

45. de Weerdt I, Hofland T, de Boer R, Dobber JA, Dubois J, van Nieuwenhuize D, et al. Distinct immune composition in lymph node and peripheral blood of CLL patients is reshaped during venetoclax treatment. Blood Adv. 2019;3(17):2642-52.

46. Kondo K, Shaim H, Thompson PA, Burger JA, Keating M, Estrov Z, et al. Ibrutinib modulates the immunosuppressive CLL microenvironment through STAT3-mediated suppression of regulatory Bcell function and inhibition of the PD-1/PD-L1 pathway. Leukemia. 2018;32(4):960-70.

\section{Figures}


A)

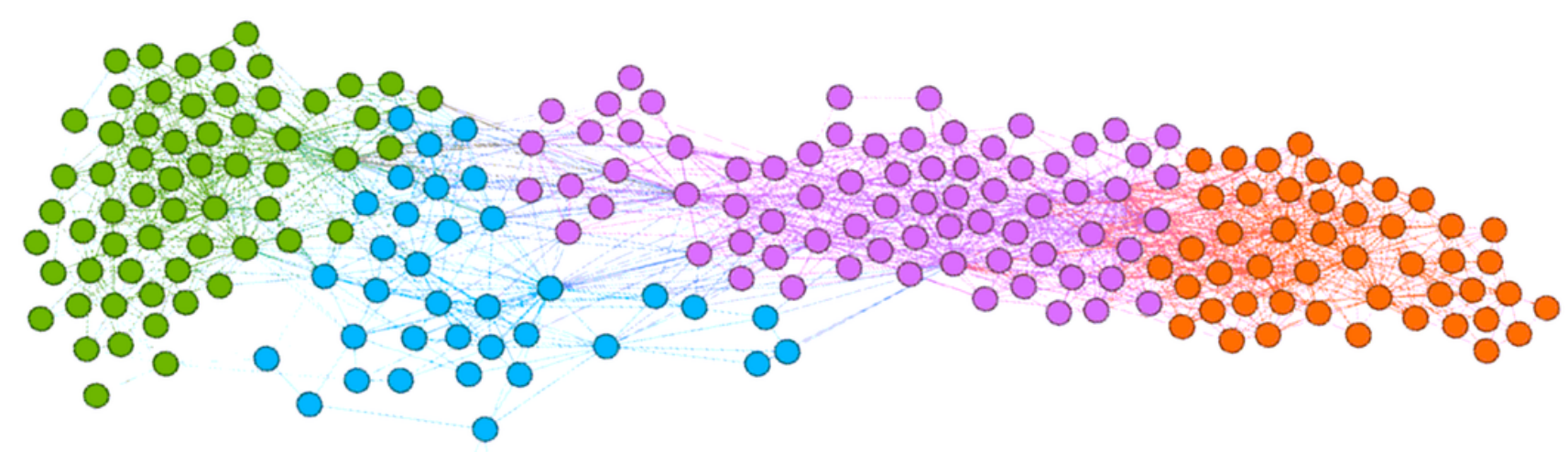

\section{$\begin{array}{llll}\text { cluster } 1 & \text { o cluster } 2 & \text { cluster } 3 & \text { cluster } 4\end{array}$}

\section{Activation}

B)

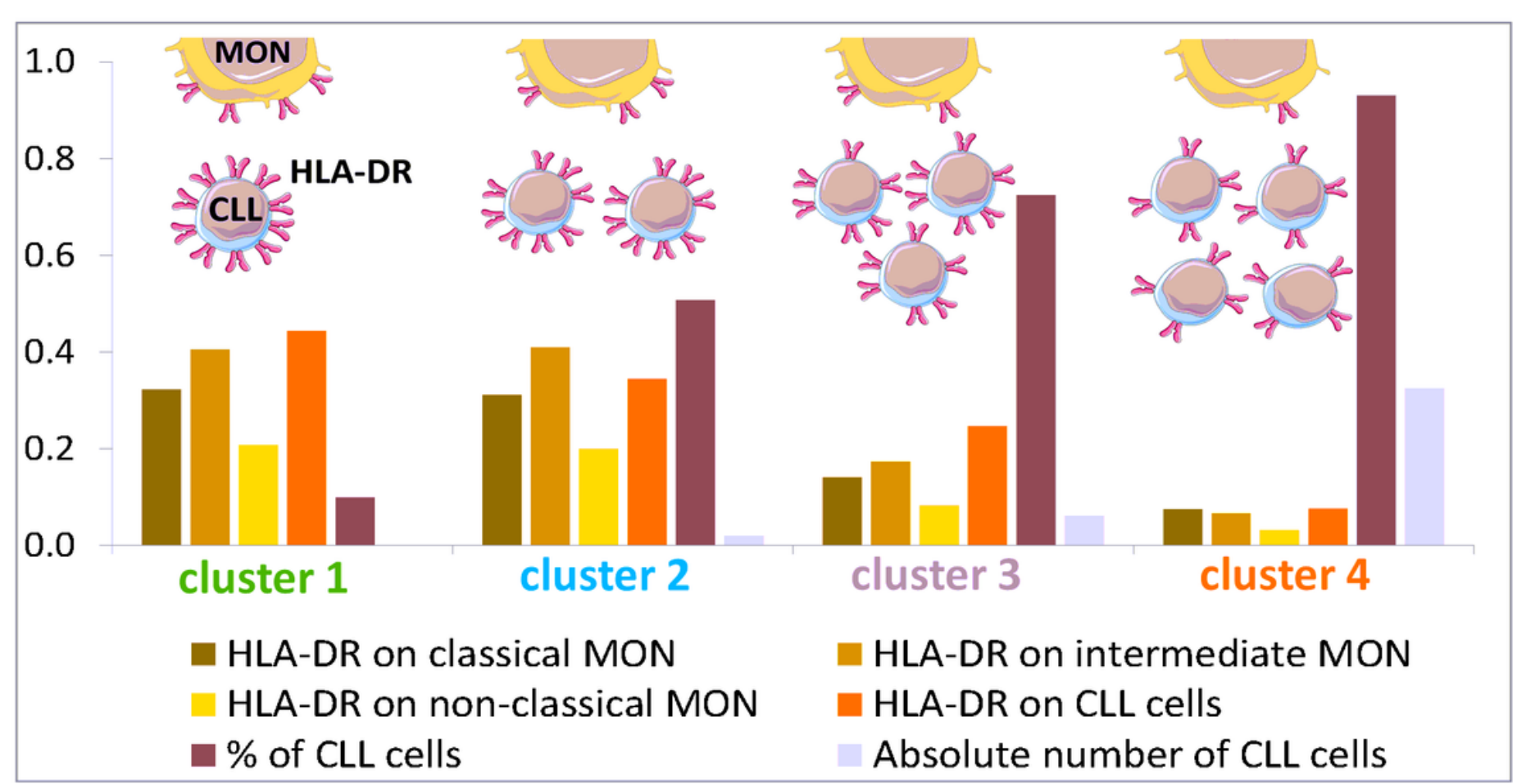

Figure 1

A) Patient similarity network (PSN) showing the distribution of CLL patients into four clusters according to percentage and CLL cell counts and expression of HLA-DR on CLL cells and subsets of monocytes (classical, intermediate, non-classical MON). Individual clusters are colored; each node corresponds to one patient; lines connect patients with the highest similarity of expression/cell count profiles. B) Characteristics of obtained clusters revealed by PSN. The y-axis in the graph shows the average values of the used markers/cell counts normalized to the maximum value in the data set. 


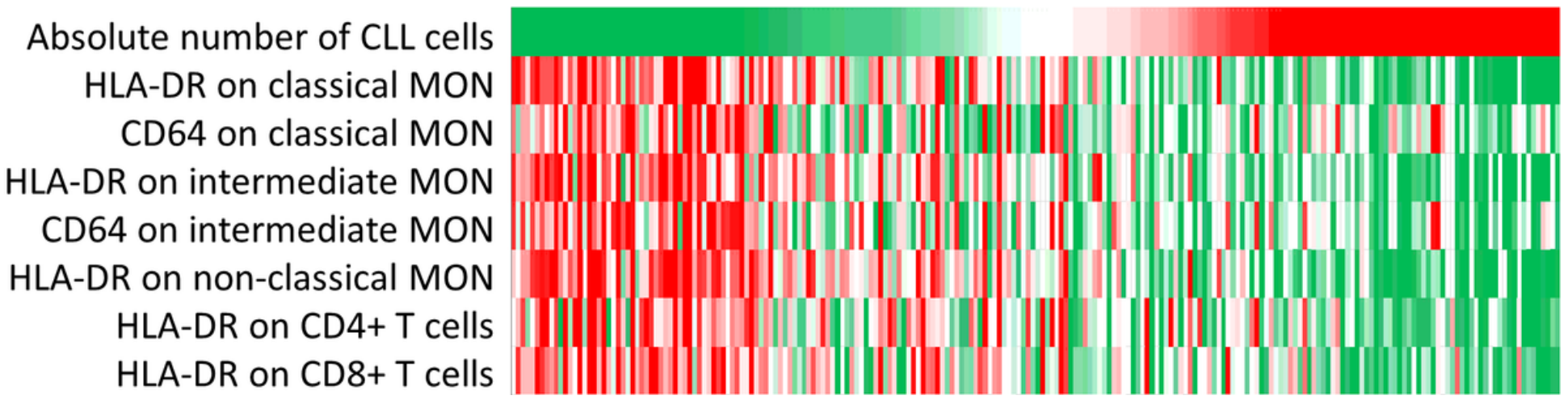

Figure 2

Heat map illustrating negative correlations of activation markers on immune cells with the CLL cell counts. Each column represents an individual CLL patient. Patients are ranked according to the CLL cell counts (x109cells/L) from the lowest (green) to the highest (red) values. The expression (MFI) of HLA-DR and CD64 on the populations were colored according to the range of values of individual populations from the lowest (green) to the highest (red) values. 
A)

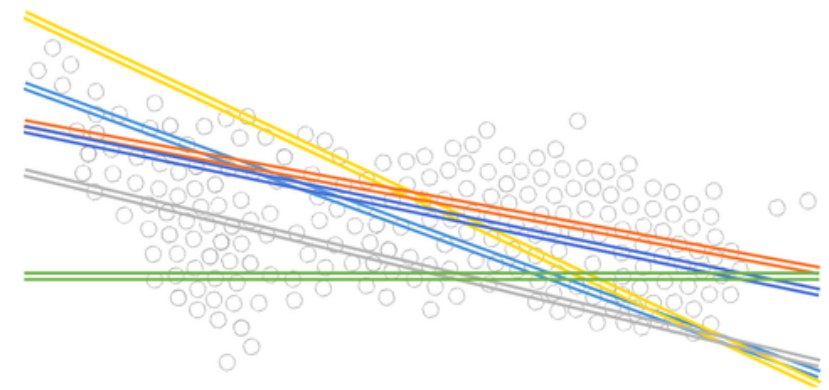

HLA-DR on

$=$ MON classical

= MON intermediate

= MON non-classical

$=\mathrm{CD} 4+$ cells

$=\mathrm{CD} 8+$ cells

C)

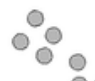

B)

\%。

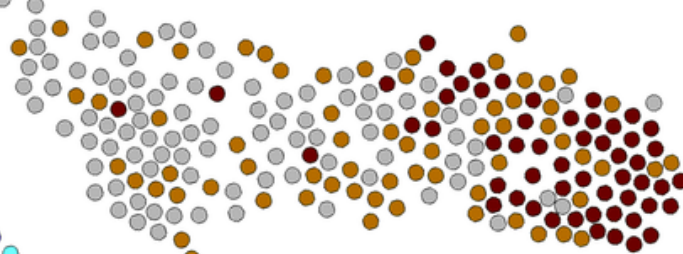

$$
=\text { NK cells }
$$

D)

$$
\because
$$

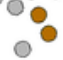

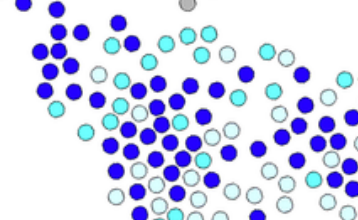

00

\%o

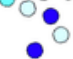

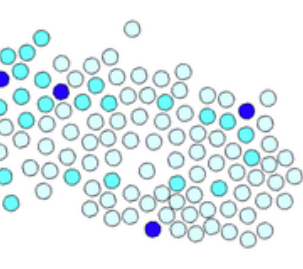

- $10-75 \times 10^{9} \mathrm{CLL}$ cells/L

- $>75 \times 10^{9} \mathrm{CLL}$ cells/L

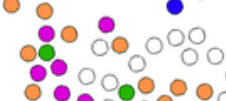
8000

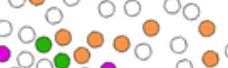

- 0800 0.90.

o

E)

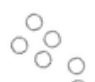

$r^{\circ}$

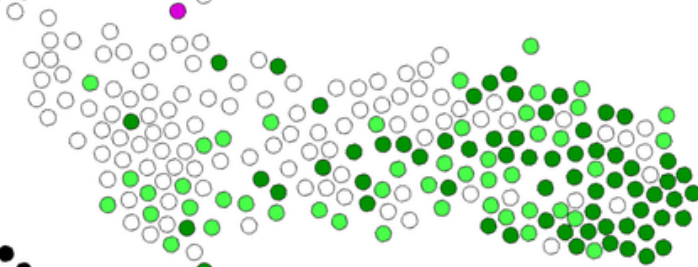

F)

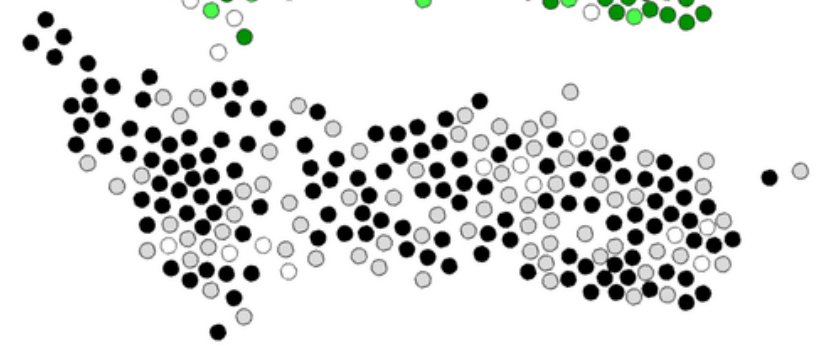

ibrutinib

- idelalisib

venetoclax

$\bigcirc$ others

treatment-naïve CLL patients who required treatment within 1 year

treatment-naïve patients

others

unmutated $/ g H V$

mutated IgHV

not available

\section{Figure 3}

The relationship between immune cell activation and clinical parameters in CLL patients. Clustering was performed using PSN on a combination of HLA-DR expression on monocytes subsets, CD4+ and CD8+ lymphocytes and NK cells. A) The trend of changes in activation of immune subpopulations across the patient clusters. The dots represent individual CLL patients colored according to B) CLL cell counts; C) 
treatment regime; D) novel drugs treatment; E) treatment requirement in treatment-naïve patients; F) IgHV mutational status.

A)
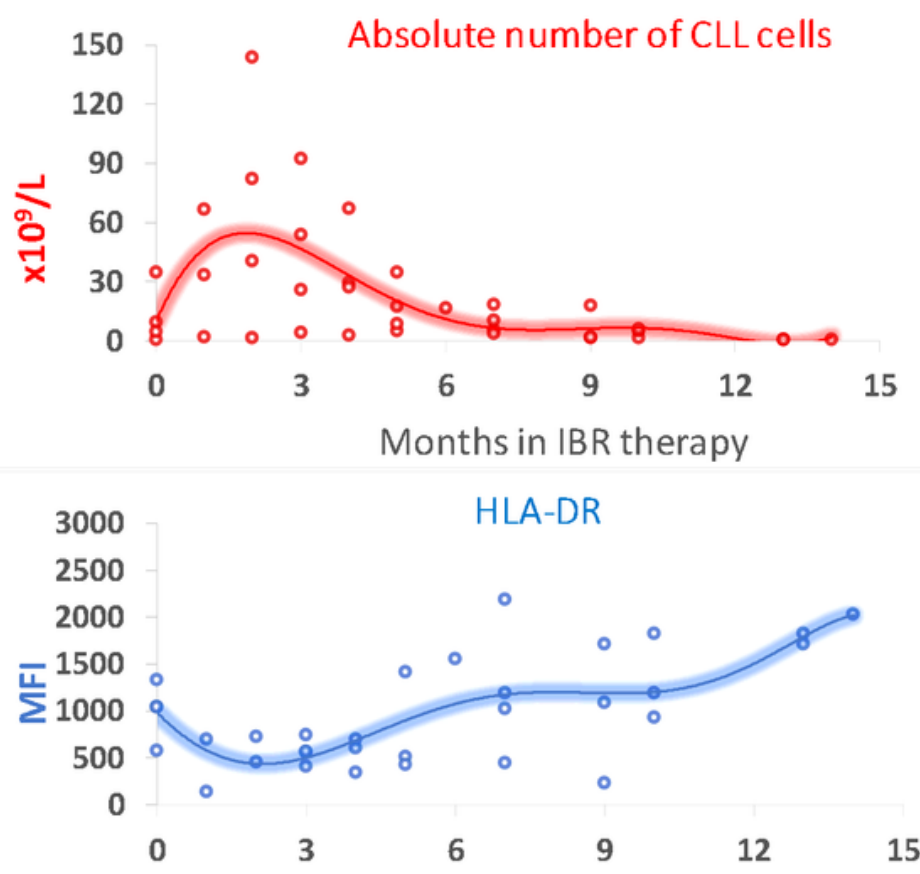

Months in IBR therapy
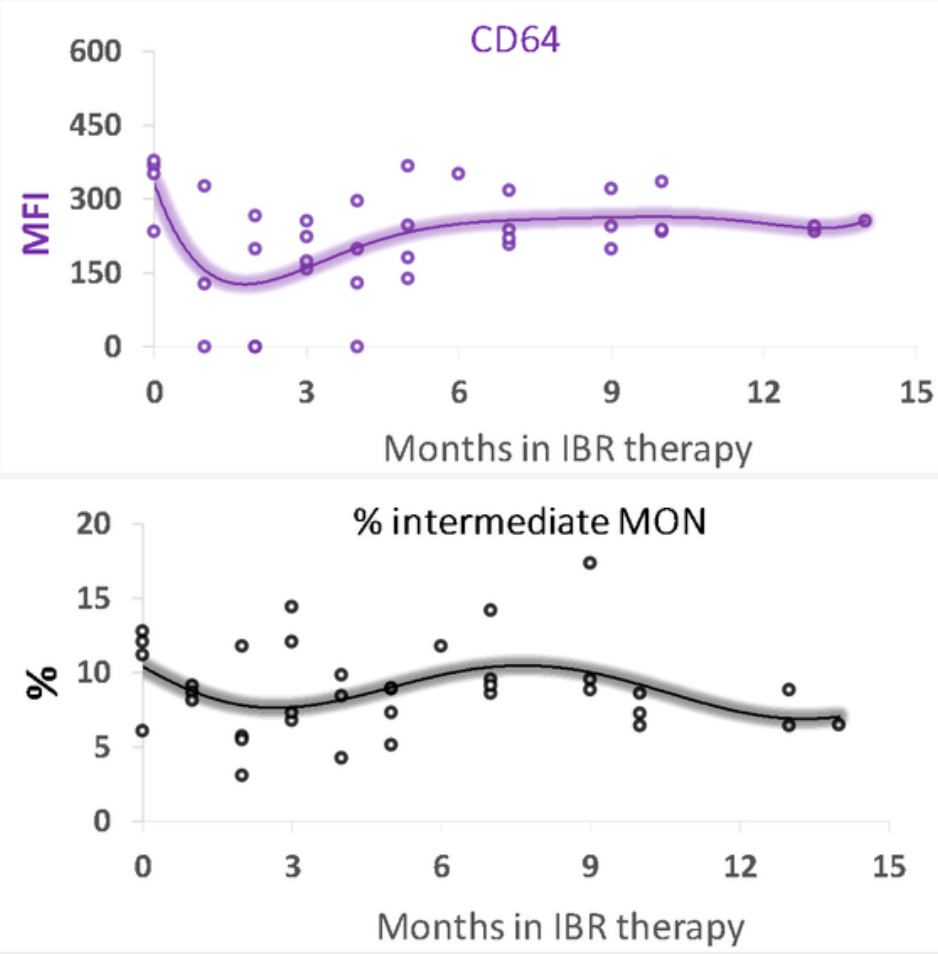

B)
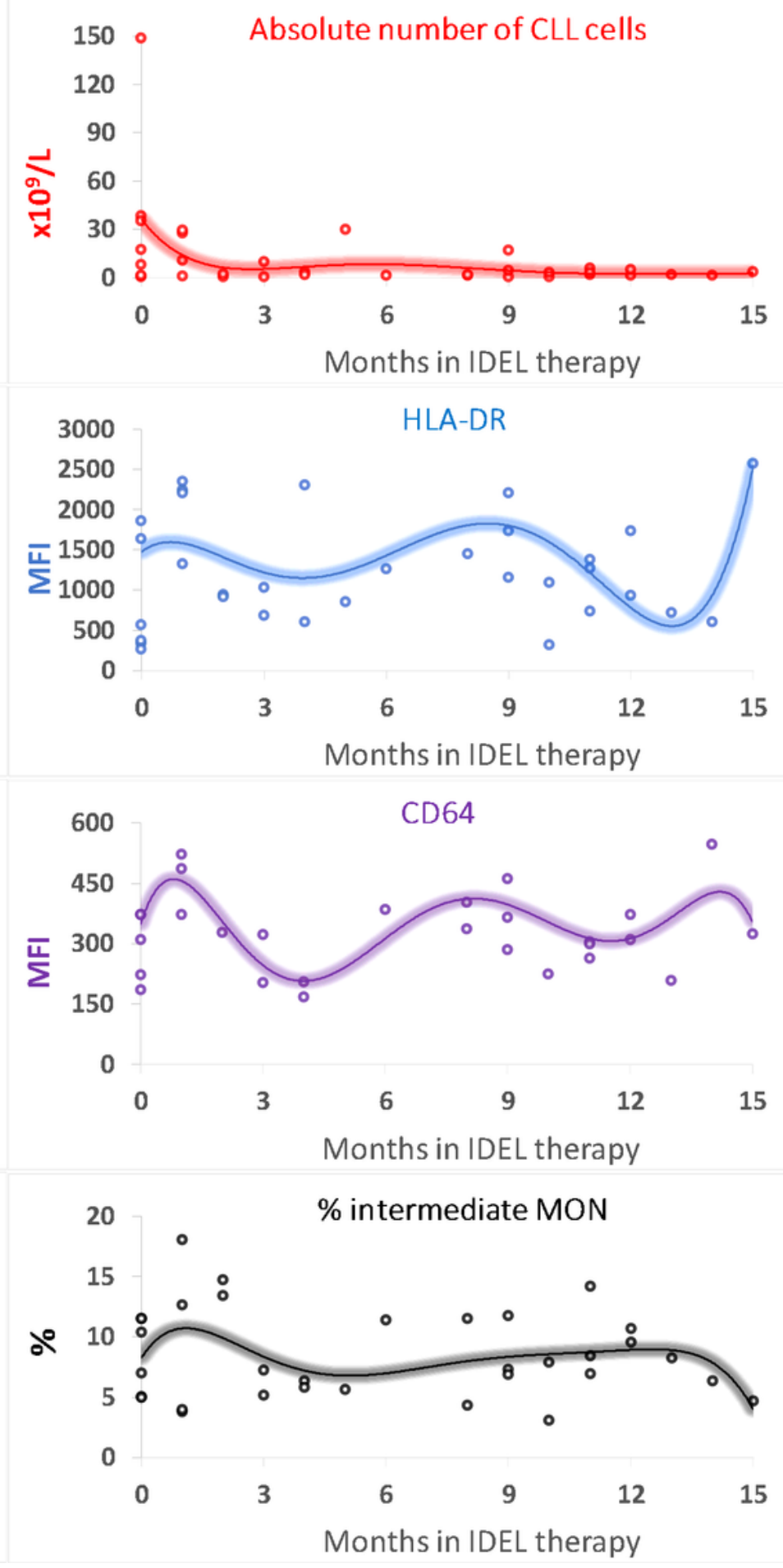

\section{Figure 4}

Time-dependent changes of CLL counts, percentages of intermediate monocytes and their expression of HLA-DR and CD64 in A) ibrutinib (IBR, n=5) and B) idelalisib (IDEL, n=6) treated patients. CLL cell counts is shown by red dots and curves (5th-degree polynomial regression), expression of HLA-DR and CD64 on 
intermediate monocytes is shown by blue and violet graphs, respectively, and percentages of intermediate monocytes is shown by black dots and curves. Some patients were not sampled at all-time points.

\section{A)}

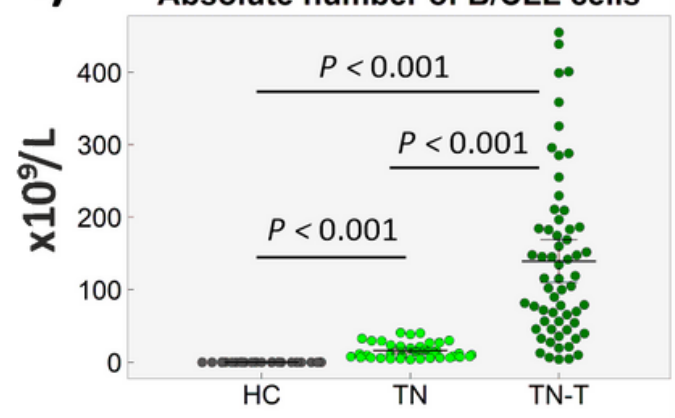

C)

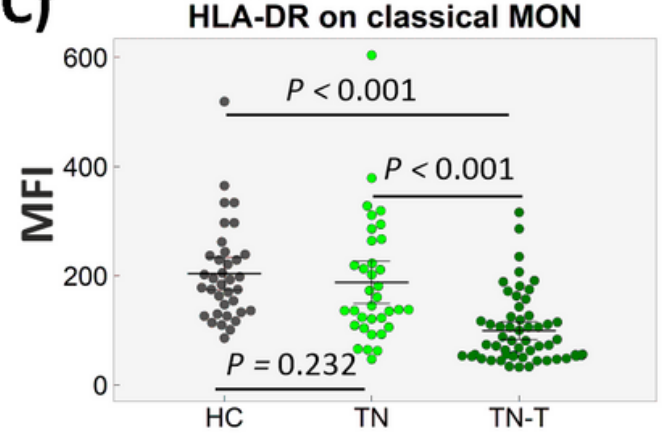

B)

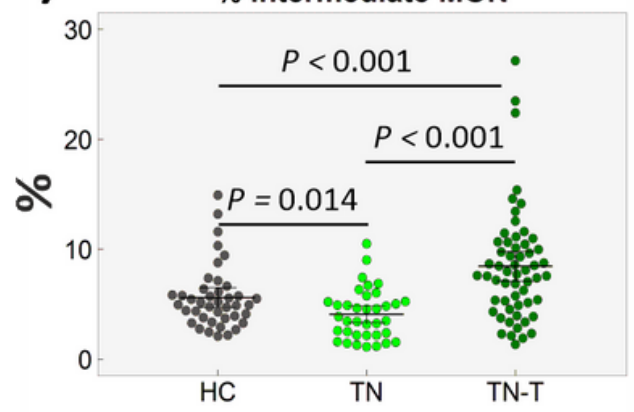

HLA-DR on intermediate MON

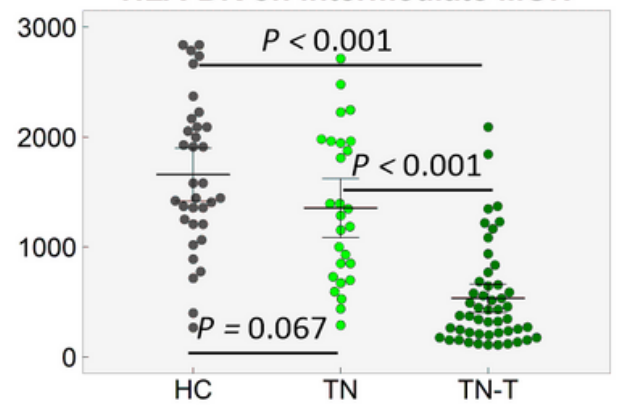

$\%$ Treg cells of CD4+ cells

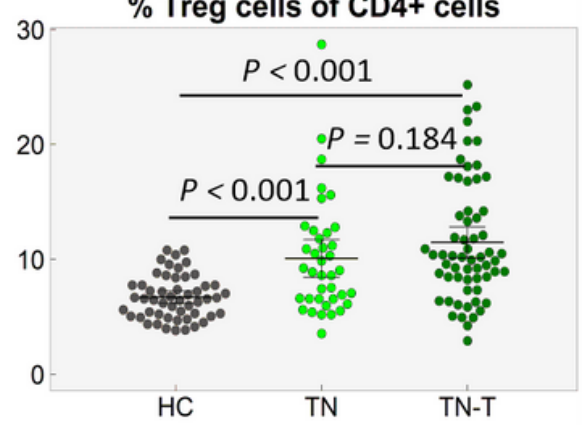

HLA-DR on non-classical MON

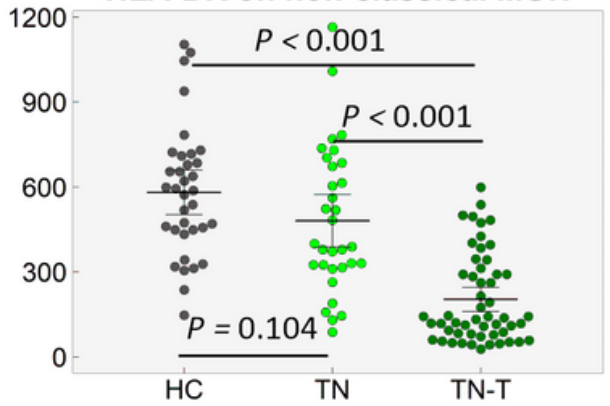

\section{Figure 5}

Distribution of circulating cells in treatment-naïve (TN) CLL patients who required treatment within 1 year (TN-T), TN patients with the indolent course (TN) and healthy controls (HC). A) CLL cell counts in CLL patients or B cells in healthy controls, B) percentages of intermediate monocytes and proportion of Treg cells within CD4+ T cells, and C) expression of HLA-DR on classical, intermediate and non-classical monocytes (MON). In some patients, values are missing due to the low proportion of some immune populations. 


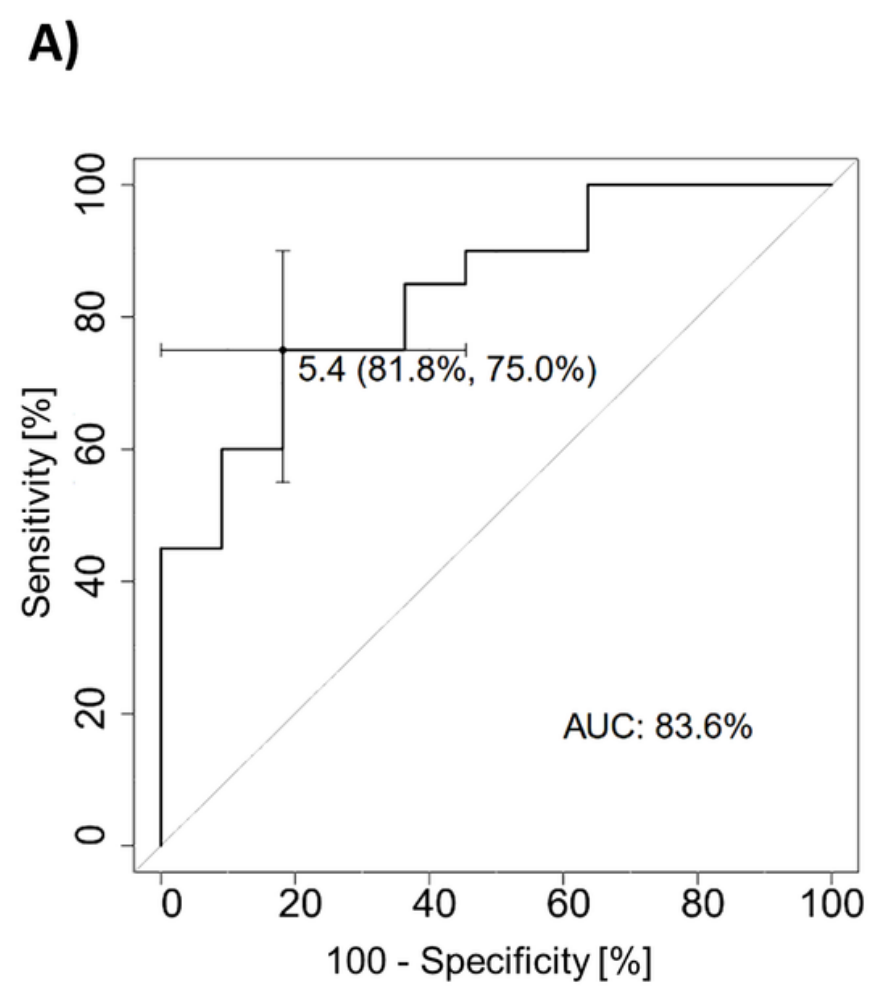

B)
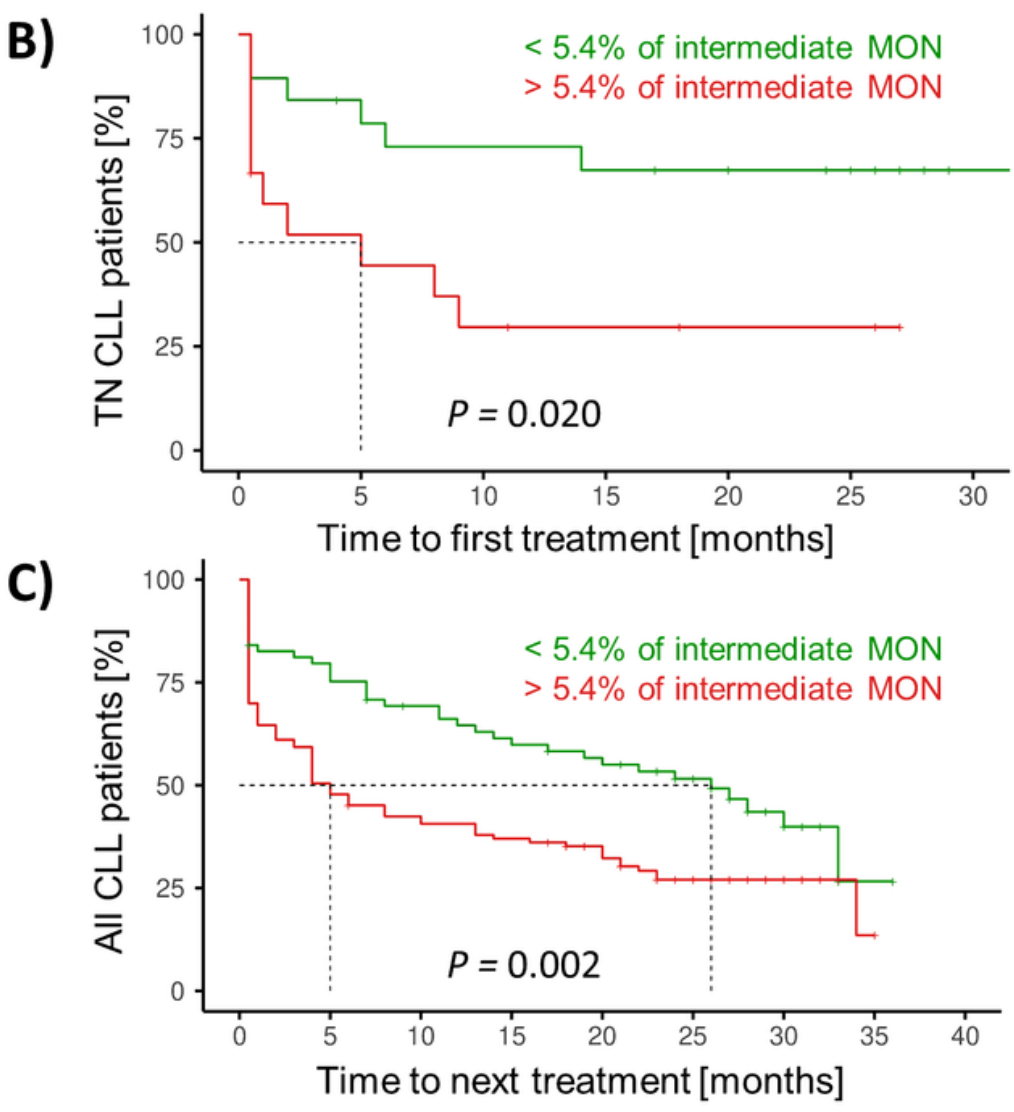

Figure 6

Prognostic potential of intermediate monocyte percentage in CLL patients. A) ROC curve for TN patients sampled at the diagnosis subdivided according to the time-to-event endpoint (TTE) during the postsampling follow-up. Kaplan-Meier curves show TTE (green curve for patients with a low percentage of intermediate MON, while red curve is for patients with more than $5.4 \%$ of intermediate MON) in groups of B) newly diagnosed TN patients and C) validation cohort of CLL patients in all stages of the disease.

\section{Supplementary Files}

This is a list of supplementary files associated with this preprint. Click to download.

- MikulkovaJTRANSLMEDSuppl.docx 